\title{
Publisher Correction: Whole genome sequencing in psychiatric disorders: the WGSPD consortium
}

\author{
Stephan J. Sanders, Benjamin M. Neale (D), Hailiang Huang (D), Donna M. Werling, Joon-Yong An (D), Shan Dong, \\ Whole Genome Sequencing for Psychiatric Disorders (WGSPD), Goncalo Abecasis, P. Alexander Arguello (D, \\ John Blangero, Michael Boehnke, Mark J. Daly (D, Kevin Eggan, Daniel H. Geschwind, David C. Glahn, David B. Goldstein, \\ Raquel E. Gur, Robert E. Handsaker (D), Steven A. McCarroll, Roel A. Ophoff (D, Aarno Palotie, Carlos N. Pato, \\ Chiara Sabatti, Matthew W. State, A. Jeremy Willsey, Steven E. Hyman (D, Anjene M. Addington (D), Thomas Lehner and \\ Nelson B. Freimer (D)
}

Correction to: Nature Neuroscience https://doi.org/10.1038/s41593-017-0017-9 (2017), published online 28 November 2017.

In the version of this article initially published, the consortium authorship and corresponding authors were not presented correctly. In the PDF and print versions, the Whole Genome Sequencing for Psychiatric Disorders (WGSPD) consortium was missing from the author list at the beginning of the paper, where it should have appeared as the seventh author; it was present in the author list at the end of the paper, but the footnote directing readers to the Supplementary Note for a list of members was missing. In the HTML version, the consortium was listed as the last author instead of as the seventh, and the line directing readers to the Supplementary Note for a list of members appeared at the end of the paper under Author Information but not in association with the consortium name itself. Also, this line stated that both member names and affiliations could be found in the Supplementary Note; in fact, only names are given. In all versions of the paper, the corresponding author symbols were attached to A. Jeremy Willsey, Steven E. Hyman, Anjene M. Addington and Thomas Lehner; they should have been attached, respectively, to Steven E. Hyman, Anjene M. Addington, Thomas Lehner and Nelson B. Freimer. As a result of this shift, the respective contact links in the HTML version did not lead to the indicated individuals. The errors have been corrected in the HTML and PDF versions of the article.

Published online: 16 March 2018

https://doi.org/10.1038/s41593-018-0102-8

\section{Publisher Correction: Single-cell analysis of experience-dependent transcriptomic states in the mouse visual cortex}

Sinisa Hrvatin, Daniel R. Hochbaum (D, M. Aurel Nagy, Marcelo Cicconet, Keiramarie Robertson, Lucas Cheadle, Rapolas Zilionis, Alex Ratner, Rebeca Borges-Monroy, Allon M. Klein, Bernardo L. Sabatini (D) and Michael E. Greenberg (D)

Correction to: Nature Neuroscience https://doi.org/10.1038/s41593-017-0029-5, published online 11 December 2017.

In the version of this article initially published, the $x$-axis labels in Fig. $3 \mathrm{c}$ read Vglut, Gad1/2, Aldh1l1 and Pecam1; they should have read $\mathrm{Vglut}^{+}, \mathrm{Gad} 1 / 2^{+}$, Aldh $1 \mathrm{ll} 1^{+}$and Pecam $1^{+}$. In Fig. 4, the range values were missing from the color scales; they are, from left to right, $4-15,0-15,4-15$ and $0-15$ in Fig. $4 \mathrm{a}$ and $4-15,4-15$ and $4-8$ in Fig. 4 h. In the third paragraph of the main text, the phrase reading "Previous approaches have analyzed a limited number of inhibitory cell types, thus masking the full diversity of excitatory populations" should have read "Previous approaches have analyzed a limited number of inhibitory cell types and masked the full diversity of excitatory populations." In the second paragraph of Results section "Diversity of experience-regulated ERGs," the phrase reading "thus suggesting considerable divergence within the gene expression program responding to early stimuli" should have read "thus suggesting considerable divergence within the early stimulus-responsive gene expression program." In the fourth paragraph of Results section "Excitatory neuronal LRGs," the sentence reading "The anatomical organization of these cell types into sublayers, coupled with divergent transcriptional responses to a sensory stimulus, suggested previously unappreciated functional subdivisions located within the laminae of the mouse visual cortex and resembling the cytoarchitecture in higher mammals" should have read "The anatomical organization of these cell types into sublayers, coupled with divergent transcriptional responses to a sensory stimulus, suggests previously unappreciated functional subdivisions located within the laminae of the mouse visual cortex, resembling the cytoarchitecture in higher mammals." In the last sentence of the Results, "sensory-responsive genes" should have read "sensory-stimulus-responsive genes." The errors have been corrected in the HTML and PDF versions of the article. 